\title{
Paramétrisation de l'énergie des vagues en zone de surf interne : Profil de plage « plane» et profil de plage à barres
}

\author{
Nadia Sénéchal $^{(\mathrm{a})}$, Philippe Bonneton ${ }^{(\mathrm{b})}$, Hélène Dupuis ${ }^{(\mathrm{b})}$ \\ ${ }^{a}$ Maître de Conférences, Département de Géologie et Océanographie, Université de Bordeaux I, avenue des \\ Facultés, 33405 Talence, cedex. + 33(0)5 40002450. \\ n.senechal@epoc.u-bordeauxl.Fr \\ ${ }^{\mathrm{b}}$ Chargé de recherche, UMR 5805-EPOC, Université de Bordeaux I, avenue des Facultés, 33405 Talence, \\ cedex. \\ h.dupuis@epoc.u-bordeaux1.fr.p.bonneton@epoc.u-bordeauxl.Fr
}

\section{Résumé}

Le paramètre défini comme le rapport entre la hauteur significative des vagues et la profondeur d'eau est un paramètre clé dans de nombreux modèles paramétriques en ingénierie mais également de long terme de comportement. Ce paramètre a longtemps été considéré comme constant dans la zone de surf interne. L'objectif de cette étude est de montrer la variabilité de ce paramètre et d'analyser les paramètres adimensionnés susceptibles de contrôler son évolution dans la zone de surf dans le cas d'une plage "plane » (ne présentant pas de replats ou de systèmes de barres) mais également dans le cas d'une plage à barres.

\section{$\underline{\text { Abstract }}$}

The parameter defined as the ratio of significant wave height to mean water depth is a key parameter in parametric wave models and much effort has been devoted to investigate its evolution in the surf zone. Indeed, it has been long believed that this parameter is constant in the inner surf zone. In this paper, we show the variability of this parameter in the inner surf zone and then, we examine the parameters, which control the evolution in the surf zone of both a linear and a barred beach.

Mots clés : approche in situ, paramètre

\section{Introduction}

La zone de surf correspond à la zone où l'énergie des vagues est dissipée, parfois très rapidement (sur quelques dizaines de mètres). La connaissance de l'évolution de la hauteur des vagues en zone de surf est un préalable indispensable à la connaissance de la dynamique en zone de surf. En effet, la hauteur des vagues permet d'estimer l'énergie potentielle associée à un front d'onde (et cinétique si on se place dans la théorie linéaire). En connaissant l'évolution de la hauteur des vagues en zone de surf, on peut appréhender le phénomène de dissipation d'énergie liée au déferlement des vagues mais on peut également estimer les variations du niveau moyen du plan d'eau (set-up et set-down) et la circulation en zone de surf. Ainsi ces dernières décennies, ce point a fait l'objet de nombreuses études expérimentales (in situ ou en laboratoire) mais également de développement de modèles paramétriques, analytiques et numériques.

Les données expérimentales in situ $\left[{ }^{1},{ }^{2},{ }_{3}^{3}\right]$ et en laboratoire $\left[{ }^{4}, 5\right]$ ont généralement mis en évidence une tendance linéaire entre la hauteur des vagues et la profondeur d'eau locale en zone de surf interne. 
Ainsi la première idée qui a été très largement répandue était que la hauteur des vagues en zone de surf interne est contrôlée par la profondeur d'eau locale. En fait dès le début des années 1960, les études ont portées sur les valeurs du paramètre adimensionné défini comme le rapport entre la hauteur des vagues $\mathrm{H}$ (hauteur moyenne, significative...suivant les auteurs et le type de houle étudiée : aléatoire ou régulière) et la profondeur d'eau locale d:

$$
\gamma=\frac{H}{d}
$$

Ce rapport a longtemps été considéré comme constant dans la zone de surf et égal à sa valeur au point de déferlement. Ainsi en ingénierie, la valeur 0.78 établie théoriquement par McCowan ${ }^{6}$ pour une onde solitaire sur plage linéaire, a longtemps été utilisée.

Dès les années 1960, les résultats expérimentaux de Horikawa et $\mathrm{Kuo}^{7}$, Nakamura et al. ${ }^{8}$, Street et Camfield ${ }^{9}$ et Divoky et al. ${ }^{10}$ ont montré que l'hypothèse d'un rapport constant dans la zone de surf n'était pas valable. Les données expérimentales de Horrikawa et $\mathrm{Kuo}^{7}$, indiquent que le rapport décroît d'une valeur proche de 0.8 au point de déferlement jusqu'à atteindre une valeur à peu près constante qui se situe autour de 0.5 en zone de surf interne. Par la suite, les travaux en laboratoire de Stive ${ }^{4}$ puis de Svendsen ${ }^{5}$ ont permis de mettre en évidence qu'en réalité le rapport commençait effectivement par décroître jusqu'à atteindre un minimum dans la zone de surf interne mais qu'ensuite il recommençait à croître jusqu'à la ligne d'eau. Ces travaux sont confortés par les récents résultats de Raubenheimer et al. ${ }^{11}$ établis à partir de données in situ, qui indiquent que n'est pas constant dans la zone de surf interne. Ils mettent en évidence des valeurs se situant entre 0.2 et jusqu'à 1.4 et une augmentation de ce rapport lorsqu'on se rapproche de la ligne d'eau. De plus, les travaux de Ruessink et al. ${ }^{12}$ mettent en évidence la nécessité de réajuster ce paramètre le long d'un profil de plage à barres afin d'améliorer les performances des modèles numériques employés pour estimer l'évolution de la hauteur des vagues en zone de surf.

Dans cette étude, nous proposons, à partir de données acquises en milieu naturel et en s'appuyant sur des modèles hydrodynamiques validés de décroissance de hauteur des vagues en zone de surf, de déterminer les paramètres adimensionnés qui contrôlent l'évolution de ce rapport en zone de surf interne.

\section{$\underline{\text { 2.Résultats et discussions }}$}

\subsection{Méthode}

Cette étude s'appuie sur les données hydrodynamiques de deux campagnes de mesures qui se sont déroulées sur le site atelier du Truc Vert (Côte Girondine) dans le cadre du Programme National Environnements Côtiers. L'une s'est déroulée en mai $1998\left[^{2}\right]$ en présence d'une mer du vent peu énergétique $\left(\mathrm{H}_{\mathrm{s}}<1.2 \mathrm{~m}\right.$ et $\left.\mathrm{T} \approx 6.5 \mathrm{~s}\right)$, les capteurs avaient été déployés le long d'une ligne cross-shore en dehors de l'influence du système barre-baïne. L'autre campagne s'est déroulée en Octobre $2001\left[^{13}\right]$ en présence d'une houle longue régulière énergétique $\left(1.7 \mathrm{~m}<\mathrm{H}_{\mathrm{s}}<2.7 \mathrm{~m}\right.$ et $\left.\mathrm{T} \approx 11-14 \mathrm{~s}\right)$, trois lignes de capteurs avaient été déployées le long d'un système barre-baïne.

Dans cette étude, nous avons retenus 5 paramètres susceptibles de contrôler l'évolution du paramètre en zone de surf :

la cambrure au large : $\frac{H_{0}}{L_{0}}$,

le rapport au point de déferlement (ou au début de la zone de surf interne) $: \gamma_{b}=\frac{H_{b}}{d_{b}}$, 
le paramètre de dispersion du train de vagues : $\mu=\frac{d}{L_{0}}$,

la distance de la zone de surf adimensionnée par la longueur d'onde au large $: \frac{\Delta x}{L_{0}}$, que nous appellerons largeur relative de la zone de surf,

et la pente de plage :

\subsection{Evolution du rapport dans la zone de surf interne}

La figure 1 illustre l'évolution du rapport en fonction de la profondeur d'eau dans la zone de surf interne. Les données correspondent aux mesures effectuées en Octobre 2001 en présence d'une houle longue régulière énergétique.

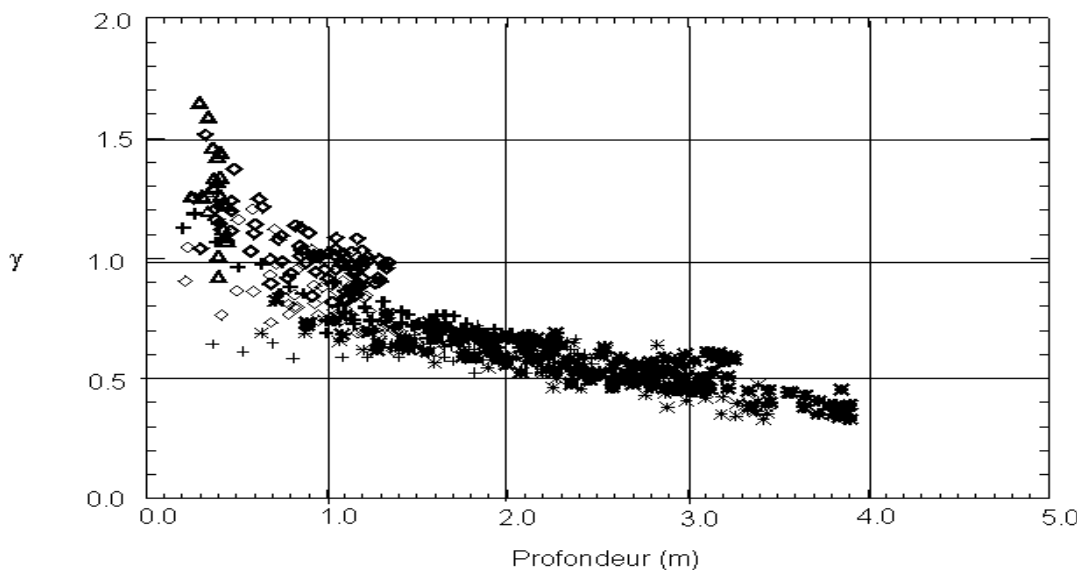

Figure 1. Evolution du rapport $\gamma$ en fonction de la profondeur dans la zone de surf interne

Cette figure met clairement en évidence que le rapport n'est pas constant dans la zone de surf mais qu'il croit lorsqu'on se rapproche de la ligne d'eau jusqu'à atteindre des valeurs proches de 1.5. Ces résultats sont aussi bien en accord avec ceux précédemment établis in situ par Raubenheimer et al. ${ }^{11}$ qu'avec ceux établis en laboratoire par Stive ${ }^{4}$ et Svendsen ${ }^{5}$.

Dans le paragraphe suivant, nous nous placerons d'abord dans le cas d'une plage " plane », c'est-à-dire dans le cas d'une plage ne présentant pas de systèmes barre-baïne et nous nous limiterons aux cas où la période des vagues peut être considérée comme constante le long de l'axe de propagation de la vague (variations $<5 \%$ ).

\subsection{Cas d'une plage « plane »}

Dans le cas d'une plage «plane», le nombre de paramètres peut être ramené à 4. La largeur relative de la zone de surf peut en effet être estimée à partir de la profondeur d'eau locale et de la pente de plage. Parmi ces 4 paramètres, nous avons pu évaluer l'impact de trois paramètres à partir des données acquises sur le terrain :

la cambrure au large : $\frac{H_{0}}{L_{0}}$,

le paramètre de dispersion du train de vagues : $\mu=\frac{d}{L_{0}}$,

le rapport au point de déferlement (ou au début de la zone de surf interne) $: \gamma_{b}=\frac{H_{b}}{d_{b}}$. 
La figure 2 illustre l'évolution du rapport en zone de surf sur une plage «plane» en fonction de la profondeur pour deux différentes cambrures. Les données correspondent aux journées des 17 (symboles noires) et 18 Octobre (symboles gris) 2001. Les cambrures au large associées à ces deux journées étaient respectivement 0.007 et 0.011 . Cette figure met clairement en évidence que les valeurs du rapport observées en zone de surf interne sont indépendantes de la cambrure au large. Il n'est donc pas nécessaire, à priori, de prendre en compte ce paramètre pour définir une paramétrisation du rapport en zone de surf interne.

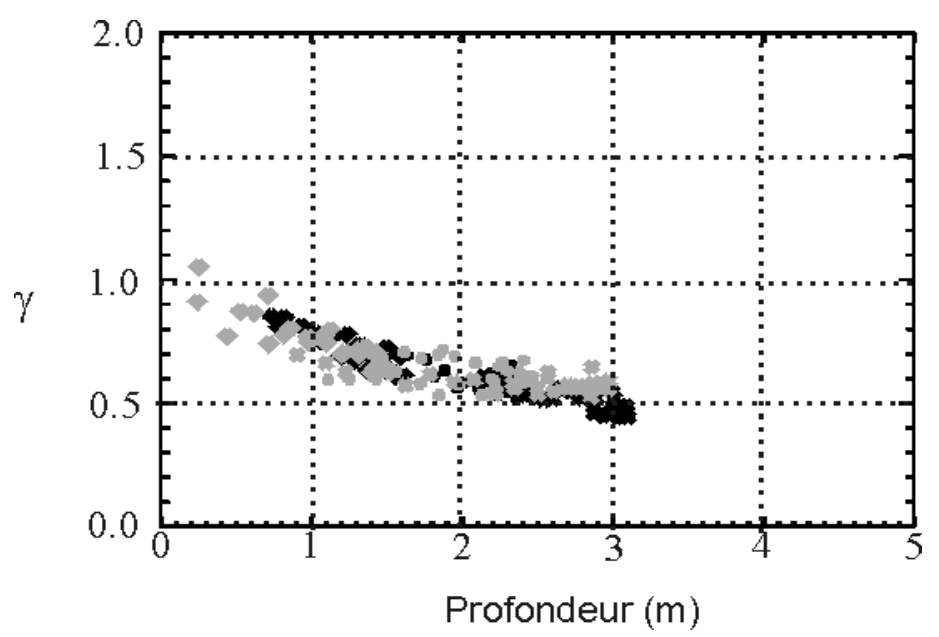

Figure 2 : Evolution du rapport en fonction de la profondeur pour deux différentes cambrures au large (0.007 en noir et 0.011 en gris)

La figure 3 met quant à elle en évidence l'influence du paramètre de dispersion $\mu=\frac{d}{L_{0}}$.

Elle a été obtenue à partir des données de Mai 1998 (en gris sur la figure) pour lesquelles la longueur d'onde au large $\mathrm{L}_{0}$ était de l'ordre de $66 \mathrm{~m}$ et à partir des données d'Octobre 2001 (en noir sur la figure) pour lesquelles la longueur d'onde au large $\mathrm{L}_{0}$ était de l'ordre de $244 \mathrm{~m}$. On observe que les deux jeux de données mettent en évidence une tendance linéaire entre la hauteur significative et la profondeur mais que la pente de la régression linéaire n'est pas la même. Sénéchal et al. ${ }^{3}$ ont pu montrer que cet écart était lié au paramètre de dispersion.

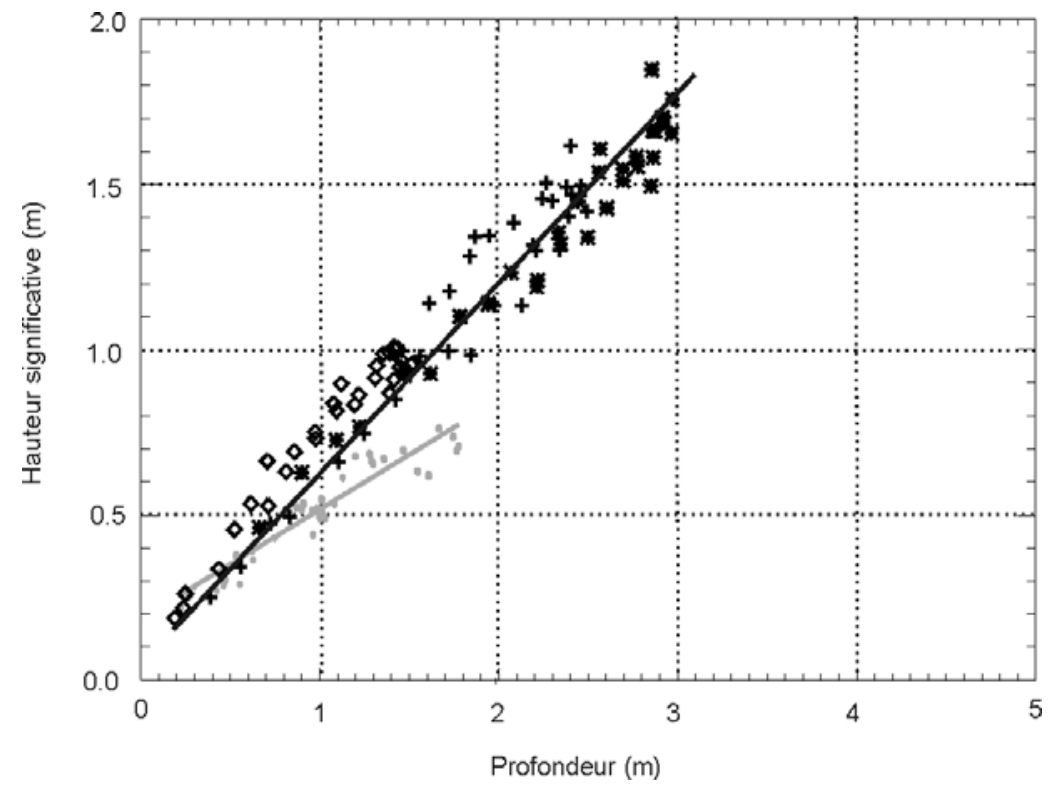

Figure 3 : Evolution de la hauteur significative en fonction de la profondeur en zone de surf interne pour deux paramètres de dispersion différents : en gris : $L_{0}=$ $66 \mathrm{~m}$ et en noir : $L_{n}=244 \mathrm{~m}$. 
Concernant le rapport au point de déferlement, il ne peut être déterminé précisément en milieu naturel, nous nous sommes donc attachés à la valeur correspondant au début de la zone de surf interne. Les précédentes études et observations indiquent que cette valeur varie peu et est de l'ordre de 0.4-0.5 pour l'ensemble des sites [ $\left[{ }^{1},{ }^{11},{ }^{3}\right]$.

Enfin, concernant la pente de la plage, nous nous sommes appuyés sur les résultats du modèle développé par Bonneton ${ }^{13}$ et validé à partir de données expérimentales qui indiquent que la pente de plage est un paramètre déterminant mais que les variations observées sur notre site ne sont pas suffisantes pour en apprécier l'impact.

\subsection{Cas d'une plage à barre}

Dans le cas d'une plage à barre, nous retrouverons les mêmes paramètres que ceux trouvés dans le cas d'une plage "plane », en dehors de la pente moyenne de la plage qui n'a dans ce cas là plus de sens. La largeur relative de la zone de surf parait par contre être un paramètre clé comme illustré par la figure 4. Cette figure représente la hauteur significative des vagues en fonction de la profondeur pour trois lignes de capteurs : (a) une ligne située au niveau de la barre, (b) une ligne située au niveau de la sortie du chenal de baïne et (c) une ligne située en dehors du système barre-baïne, sur une partie de plage «plane».
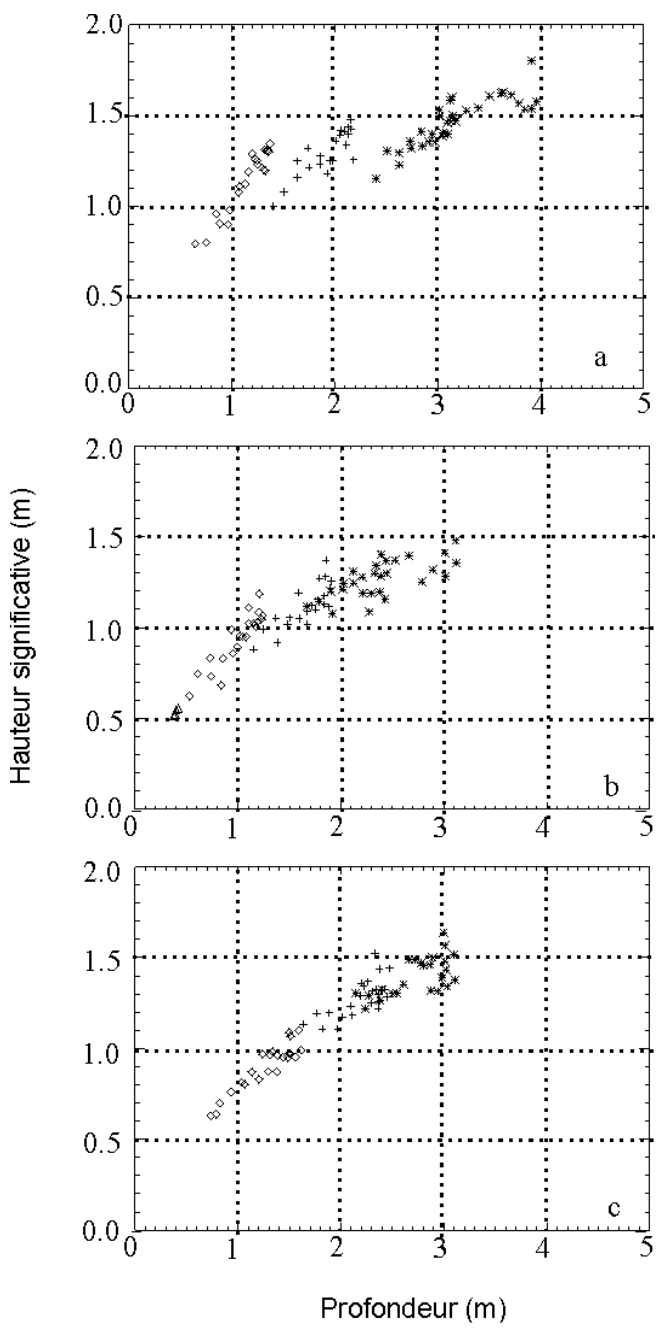

Figure 4: Hauteur significative des vagues en fonction de la profondeur : (a) profil de plage à barre, (b) sortie du chenal de baïne et (c) plage linéaire 
On distingue très nettement qu'il y a une discontinuité ente les données des différents capteurs pour la ligne située au niveau de la barre (ligne a). Sénéchal et al. ${ }^{3}$ ont pu montrer que cette discontinuité n'était pas liée à une modification du paramètre de dispersion des vagues au passage de la barre. En effet, l'analyse vague à vague du train d'ondes ne met pas en évidence de dédoublement du nombre de fronts d'ondes au passage de la barre et de modification significative des périodes. Ces discontinuités seraient liées aux variations de la largeur relative de la zone de surf avec les variations du niveau moyen du plan d'eau liées à la marée.

\subsection{Paramétrisation dans le cas d'une plage «plane» avec conservation de la période}

Nous nous sommes appuyés sur les précédents résultats de Raubenheimer et al. ${ }^{11}$ qui s'étaient placés dans le cas où la période des vagues variait pas ou peu le long d'une ligne «cross-shore». Ils ont ainsi pu remplacer dans le paramètre de dispersion, la longueur d'onde au large $\mathrm{L}_{0}$ par le nombre d'onde local. Ils ont montré qu'il existait une relation affine entre le rapport et le terme $/ \mathrm{kd}$ (où est la pente locale, $\mathrm{k}$ le nombre d'onde calculé localement et d la profondeur). Cette grandeur peut être assimilée à une quantification de la variation de profondeur sur une distance égale à une longueur d'onde.

La fréquence caractéristique retenue pour calculer le nombre d'onde est la fréquence centroïdale calculée au niveau de chaque capteur, $\mathrm{k}$ étant le nombre d'onde associé à cette fréquence et à la profondeur d'eau locale. La pente locale, est estimée sur une longueur égale à la longueur d'onde associée à la fréquence centroïdale. La figure 5 illustre les résultats de Raubenheimer et al. ${ }^{11}$ ainsi que les résultats établis à partir de nos jeux de données.

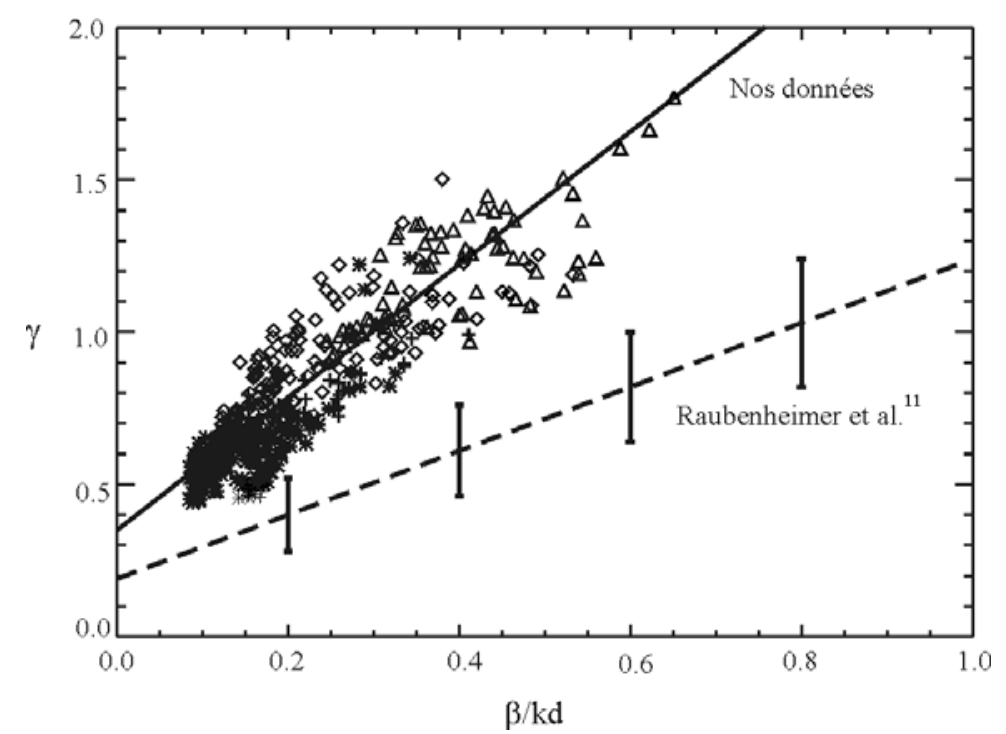

Figure 5 :en fonction de $/ k d$. Trait discontinu : résultats de Raubenheimer et al. ${ }^{11}$ [1996], trait continu : résultats des données de mai 1998, symboles : données d'Octobre 2001.

La figure 5 met clairement en évidence que nous retrouvons à partir de nos données une tendance linéaire entre le rapport et le paramètre $/ \mathrm{kd}$ en zone de surf interne, en accord avec les précédents résultats de Raubenheimer et al. ${ }^{11}$ mais avec des paramètres de pente et ordonnée à l'origine différents des leurs. Cet écart est lié d'une part au calcul de la fréquence centroïdale, en particulier au choix des fréquences de coupure pour déterminer la bande fréquentielle des vagues («sea-swell» band) mais également à la difficulté à correctement représenter l'échelle de temps caractéristique entre deux fronts d'ondes dissipant consécutifs 
dans la zone de surf où de nombreux processus peuvent modifier ce paramètre $\left[{ }^{15}\right]$. Ce dernier point avait déjà été évoqué précédemment $\left[{ }^{16}\right]$.

\section{Conclusions}

Dans cette étude, nous nous sommes intéressés au rapport défini comme le rapport entre la hauteur significative des vagues et la profondeur d'eau locale. Ce rapport est fréquemment utilisé pour paramétrer la dissipation d'énergie des vagues liée au déferlement dans de nombreux modèles numériques d'ingénierie (type sbeach, genesis...) et considéré constant dans la zone de surf. Or, dans cette étude, nous avons pu mettre en évidence, à partir de données acquises dans le milieu naturel dans le cadre du PNEC, que ce rapport n'est pas constant dans la zone de surf interne. Il dépend, dans le cas d'une plage «plane» du paramètre de dispersion et de la pente de plage et dans le cas d'une plage à barre, du paramètre de dispersion et de la largeur relative de la zone de surf. Ces résultats nous ont conduit à tester la paramétrisation proposée par Raubenheimer et al. ${ }^{11}$ pour une plage «plane». Nous retrouvons effectivement une tendance linéaire entre le rapport et le paramètre / kd mais avec des caractéristiques de pente et ordonnée à l'origine différentes. Cet écart est du d'une part à la méthode utilisée pour déterminer la bande fréquentielle des vagues («sea-swell » band) mais également à la difficulté liée à la détermination de la fréquence caractéristique des fronts déferlant en zone de surf, compte tenu que de nombreux processus interviennent à des échelles spatio-temporelles très courtes.

\section{Références}

1 Thornton E.B. and Guza R.T., (1982) Energy saturation and phase speeds measured on a natural beach. J. Geophysical Research, 87 (C12), 9499-9508.

2 Senechal N., Dupuis H., Bonneton P., et al., (2001). Observation of irregular wave transformation in the surf zone over a gently sloping sandy beach on the French Atlantic coastline. Oceanologica Acta, 24 (6), 545-556.

3 Sénéchal N., Dupuis H. and Bonneton, P., (2004). Preliminary hydrodynamic results of a field experiment on a barred beach, Truc Vert beach on October 2001. Ocean Dynamics, sous presse.

4 Stive M.J.F., (1984). Energy dissipation in waves breaking on gentle slopes. Coastal Engineering, 8, 99-127.

5 Svendsen I.A., (1984). Wave heights and set-up in a surf zone. Coastal Engineering, 8, 303-329.

6 McCowan J., (1891). On the solitary wave. Philosophical Magazine, 5eme Série, 36, 430-437.

7 Horrikawa K. and Kuo C.T., (1966). A study of wave transformation inside surf zone. Proc. Coastal Engineering Conference, 10 ${ }^{\text {th }}, \mathbf{1}, 217-233$.

8 Nakamura M., Shiraishi H. and Sasaki Y., (1966). Wave decaying due to breaking. Proc. Coastal Engineering Conference, $10^{\text {th }}, \mathbf{1}, 234-253$.

9 Street R.L. and Camfield F.E., (1966). Observations and experiments on solitary wave deformation. Proc. Coastal Engineering Conference, 10 ${ }^{\text {th }}, 1,284-293$.

10 Divoky D., Le Méhauté B. and Lin A., (1970). Breaking waves on gentle slopes. $J$. Geophysical Research, 75, 1681-1692.

11 Raubenheimer B., Guza R.T. and Elgar S., (1996). Wave transformation across the inner surf zone. J. Geophysical Research, 101, 25589-25597.

12 Ruessink B.G., Walstra D.J.R. and Southgate H.N., (2003). Calibration and verification of a parametric wave model on barred beaches. Coastal Engineering, 48, 139-149. 
13 Sénéchal N. et al., (2002). Présentation de la campagne de mesures intensives du PNEC : Truc Vert 2001. Proc VIIème Journées Nationales Génie Civil-Génie Côtier, 2, 381392.

14 Bonneton P., (2001). A note on wave propagation in the inner surf zone. C.R. Acad. Sci,. Paris 329 (S II b):27-33.

15 Sénéchal N., (2003). Etude de la propagation des vagues au-dessus d'une bathymétrie complexe en zone de surf. Thèse de l'Université de Bordeaux I, 278 p.

16 Sénéchal N., Bonneton P. and Dupuis H., (2002). Analyse de la période représentative de la houle en zone de surf. Actes des VIIème Journées Nationales Génie Côtier-Génie Civil, I, 31-38. 